\title{
STATUS OF THE INDUCTION ACCELERATION SYSTEM*
}

\author{
Y. Shimosaki ${ }^{\#}$, Y. Arakida, T. Iwashita, T. Kono, E. Nakamura, K. Takayama, M. Wake, KEK, \\ Ibaraki, Japan
}

T. S. Dixit, GUAS/AS, Ibaraki, Japan

K. Torikai, NIRS, Chiba, Japan

N. Nagura, K. Okazaki, K. Otsuka, Nippon Advanced Technology Co. Ltd., Ibaraki, Japan

\section{Abstract}

A single proton bunch confined by the barrier voltage was accelerated by the induction step-voltage from $500 \mathrm{MeV}$ to $6 \mathrm{GeV}$ at the $\mathrm{KEK} 12-\mathrm{GeV}$ proton synchrotron in March 2006. Various technical issues in the induction acceleration system, which have been faced in actual operation, are summarized.

\section{INTRODUCTION}

The induction synchrotron was proposed in 2000, in which particles are confined by a burrier voltage and accelerated by a flat voltage in the longitudinal direction [1]. The induction synchrotron was demonstrated by using the main ring of $\mathrm{KEK} 12-\mathrm{GeV}$ proton synchrotron (KEK-PS MR) in March 2006 [2]. Now, an all-ion accelerator, which is an induction synchrotron in a lower energy region, is proposed as a heavy ion driver for cancer therapy or material science [3]. Improvements of the induction acceleration devices are under way.

To generate the acceleration voltage, an induction cell is energized with a high-voltage switching power supply employing power MOS-FETs as switching elements, which is connected to a DC power supply (see Fig. 1).

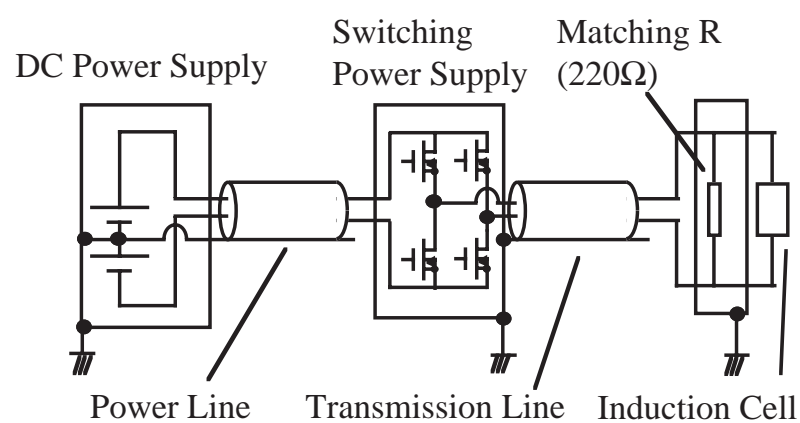

Figure 1: Equivalent circuit of induction acceleration system.

Although the demonstration of the induction synchrotron was successfully done, we have faced a lot of troubles in the induction acceleration devices, such as the DC power supply, the switching power supply, and the induction cell. In this paper, details of those troubles are summarized and status of the devices is presented, describing counter-measures or the way how we will solve the problems.

\section{INDUCTION CELL}

Ten induction cells have been installed in the tunnel of the KEK-PS MR for accelerating and confining particles. Six magnetic core troids, which are made of Finemet of Hitachi Metal, are embedded in each cell case [4]. Silicone oil with the flash point of 260 degrees centigrade was used as cooling medium. For filling the oil, a polyethylene pipe with electrical insulation and high radiation resistance has been set in the cell, which is strongly required from the structure of our cell. Since the melting point of the polyethylene is about 120 degrees centigrade, the pipe has often melted because of the low cooling efficiency for the core and the arc discharge. This means the flammable material could leak in the tunnel.

The improvement of the core-material and the case was performed from the economic point of view at that time. However, it was found after the melting accident that (1) improved core tends to be hot more than the old-one, which depends on the thickness of the Finemet ribbon and the thickness of the Finemet in the new core is $18 \mu \mathrm{m}$ and that in the old core is $13 \mu \mathrm{m}$, as shown in Fig. 2(a). In addition, (2) the flow of the oil through the new-case was not effective in comparison with the old-case (see Fig. 2(b)), even though same magnetic material was used. These reasons resulted in melting of the polyethylene pipe as shown in Fig. 3. So, the polyethylene pipe was changed to the one with higher melting point.

Even though the melting point of the polyethylene pipe becomes higher, it melted as a result of the arcing, as shown in Fig. 4. The arc discharge occurred between the case and the metal to fix the current-bar surrounding the magnetic core. At that time, not only the pipe but also the O-ring for sealing oil was damaged, and a little leak of the oil was observed. To avoid the arc discharge, this metal became roundish. This effect will be checked by a next long heat-run.

\footnotetext{
*Work supported by a Grant-In-Aid for Creative Scientific Research (KAKENHI 15GS0217).

"shimo@www-accps.kek.jp
} 

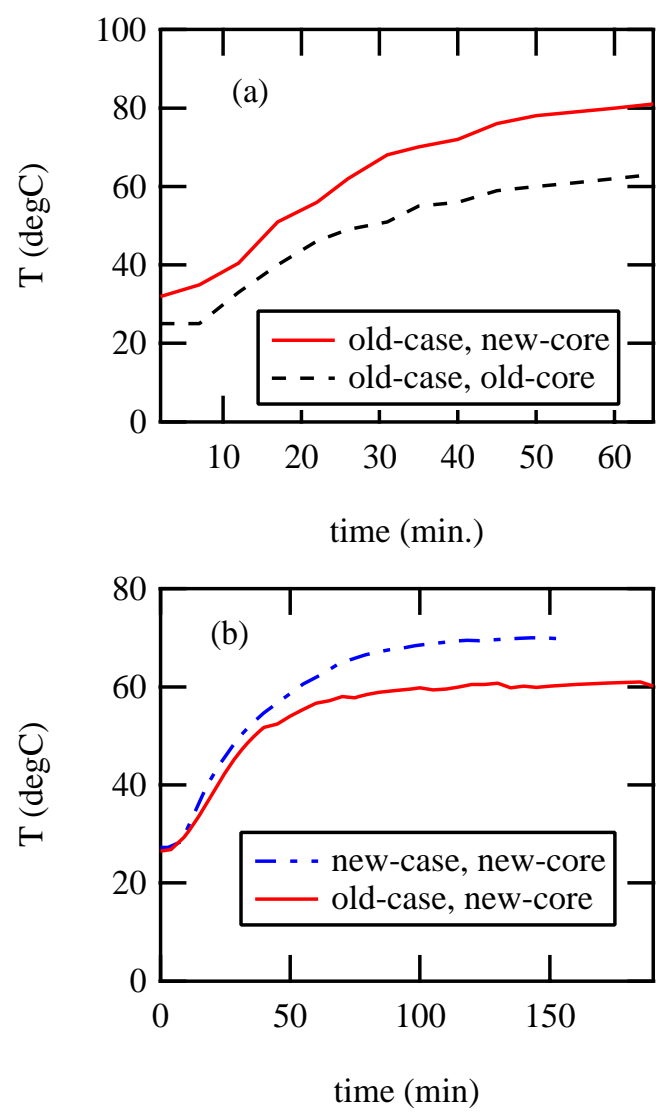

Figure 2: Temperature of polyethylene surface dependent on (a) core material and (b) structure of case.

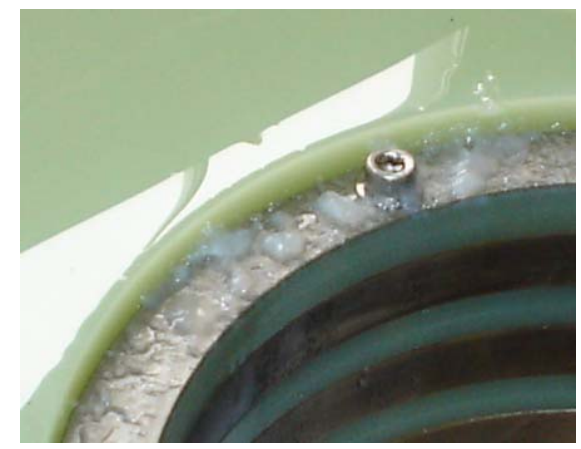

Figure 3: (white) Melted polyethylene on (thin silver) metal plate for fixing current bar around (thick silver) magnetic cores. Green is glass epoxy.

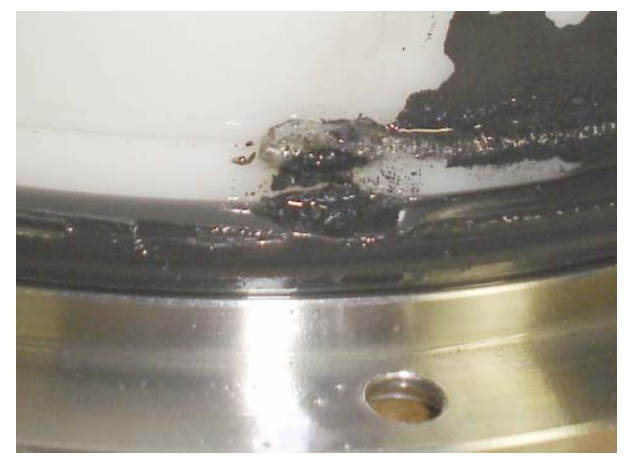

Figure 4: (white) Polyethylene pipe and (middle black) Oring melted by discharge.

\section{POWER SUPPLY AND SWICTHING POWER SUPPLY}

Six DC power supplies of $50 \mathrm{~kW} /$ maximum $3 \mathrm{kV}$ have been used to feed 10 induction acceleration systems, in which the IGBTs are utilized as switching devices in the inverter. The output voltage is delivered to the switching power supply through a high voltage power cable.

Ten switching power supplies, capable of generating a bi-polar pulse voltage at $1 \mathrm{MHz}$, have been installed in the accelerator tunnel of the KEK-PS. It consists of four switching arms, and seven MOS-FETs are connected in series in one arm [5]. Heat deposit in the MOS-FETs and their driving ICs is serious. Heat is removed by the watercooled heat-sink of a cupper block, on the surface of which the MOSFETs and driving ICs are attached.

Concerning the DC power supply, the IGBT shorted due to an over-current originated in the high frequency transformer [6], resulting in arc discharge of the switchboard. A circuit for guarding the over-current have been already set, and the high frequency transformer will be exchanged with the new one.

In the case of the switching power supply, the waterleak was often occurred from the welded spot on the copper block. Since the hydrogen embrittlement was first not taken into account, an oxygen-free copper (OFC) was not used for the material of the cupper block. It seems that the crack due to the hydrogen embrittlement arose in the material during silver-soldering, and resulted in the water leak. As the countermeasures, (1) the OFC has been chosen and another welding method was employed, and (2) the hole was made in the bottom of the switching power supply for the water not to be remained inside. The exchange of FET-board has been seldom required for the past 4 years. This indicates that the interlock system, in which over-voltage, over-current, over-temperature, and low-voltage are always watched, works well as expected. Once the interlock system is turned on, the optical signal is sent to stop the trigger and the DC power supply is turned off.

\section{NOISE PROBLEM}

Through the operation of the induction acceleration system, noise has been observed and affected other accelerator components. When the induction acceleration system was turned on, the beam intensity in the booster synchrotron (BR) of an injector for the MR became unstable, causing beam loss. A large perturbation on the excitation current of one of two back-leg coils for the COD correction was observed. This beam loss disappeared when the power supply of this back-leg coil was turned off. The control station for the BR main magnet fields and the power supplies of the back-leg coils and the DC power supply of the induction system share the same building. It was suspected that the noise originated from the induction acceleration system affected other accelerator components.

The facts: (1) the power line connecting the DC power supply and the switching power supply, which is a pair 
cable with the copper shield, passes along the control line of the BR, and (2) an antenna temporally put near the power line picked up the large signal when the induction acceleration system was turned on and the beam intensity of the BR became unstable [7], suggested the importance of direct measurement of the common mode current leaked from the power line. The result is shown in Fig. 5(a). The common mode current of $4 \mathrm{~A}$ was observed in the operation condition of $1.8 \mathrm{kV} / 15 \mathrm{~A}, 1 \mathrm{MHz}$ and the duty of $50 \%$. This common mode current was suppressed by changing the wiring throughout the induction acceleration system of Fig. 1 as shown in Fig. 5(b).

In addition, the earth current in the tunnel was measured. The current with an amplitude of $0.6 \mathrm{~A}$ was observed when the system was operated under the condition of $1.8 \mathrm{kV} / 15 \mathrm{~A}, 1 \mathrm{MHz}$ and the duty of $50 \%$. Some structure in the current profile, which corresponds to the switching frequency of the IGBT in the DC power, was found.

The noise analysis is now under way and the result will be presented in a forthcoming paper.
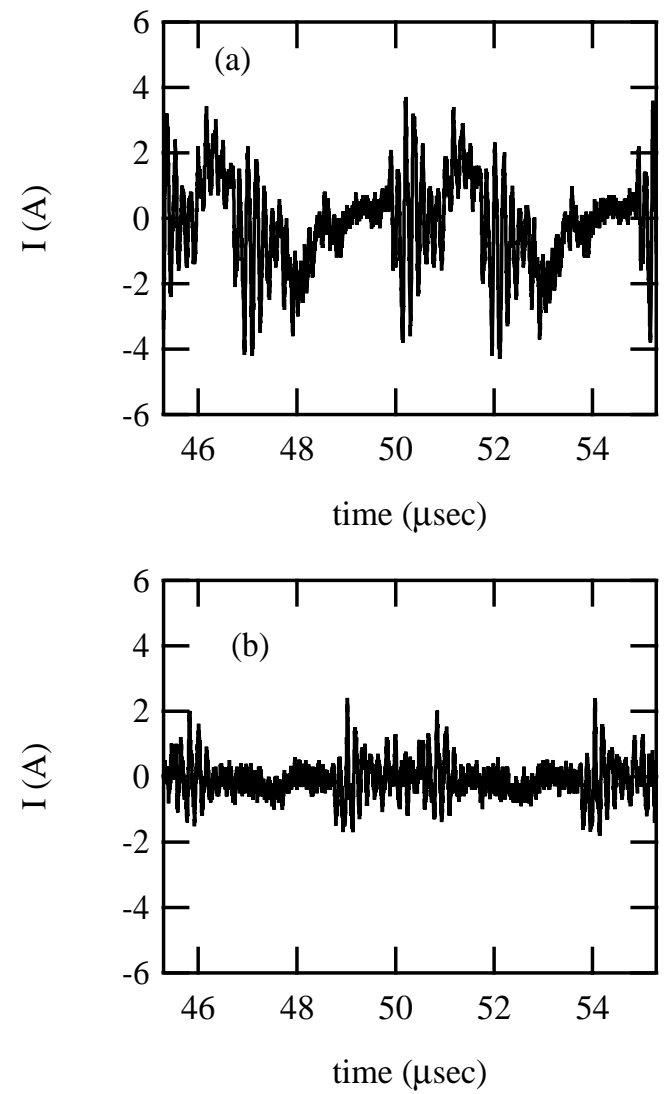

Figure 5: Common mode current passing through power line of Fig. 1.

\section{HOURS LONG HEAT RUN}

As mentioned above, the all-ion accelerator with the induction acceleration system is planned for various applications of relatively low energy heavy ions, such as cancer therapy and/or material science. The stable operation through a long time-period is required, particularly for the cancer therapy. The induction acceleration system is essentially a pulse device. Its stability in a long term has been of big concern of the public. In order to confirm the capability, three sets of the induction acceleration system were operated for 100 hours, where the conditions of $1.8 \mathrm{kV}, 1 \mathrm{MHz}$ and the duty of $50 \%$ were preliminary set.

After 3hours from the beginning, one system stopped because of the discharge mentioned above. Remained 2 systems could operate for 100 hours under the condition of $1.8 \mathrm{kV}, 1 \mathrm{MHz}$ and the duty of $50 \%$. It notes that the incorrect performance of the water gauge, which was caused by noise mentioned earlier, was observed throughout this long heat run.

After fixing the discharge, further long heat-run and the noise analysis will be required in the realistic operation mode of the all ion accelerator, where the switching frequency and the pulse length are changed in a single acceleration period.

\section{SUMMARY}

The induction synchrotron was successfully demonstrated. However, we have faced to a lot of engineering problems, which must be overcome in its actual applications. We found countermeasures for some issues. Noise problem still remains unsolved. Its effects on accelerating beam and surroundings must be more carefully studied and improved to prevent the precision devices for the future applications, particularly for the cancer therapy by the all ion accelerator, from the incorrect performance caused by the noise originated from the induction acceleration system.

\section{REFERENCES}

[1] K. Takayama and J. Kishiro, Nucl. Inst. Meth. A 451, 304 (2000).

[2] K. Takayama et al., Phys. Rev. Lett. 98, 054801 (2007). in this proceeding TUXC02.

[3] K. Takayama et al., J. Appl. Phys. 101 (2007) 063304. in this proceeding TUPAN044.

[4] K. Torikai, "High repetition rate induction cavity", the proceedings of RPIA2006 (KEK, Tsukuba), pp.70-73.

[5] K. Koseki and Y. Shimosaki, Nucl. Inst. Meth. A 556, 24 (2006). M.Wake et al., in this proceeding MOPAN042.

[6] Private communication with Nichicon (Kusatsu) corporation.

[7] Y. Shimosaki and K. Torikai, "Noise measurement concerning induction acceleration system", KEK-PS Study Report 575 (2005), written in Japanese. 